\title{
Supplementary movies
}

(Scale bars represent $5 \mu \mathrm{m}$ )

S1, S2 Generation and usage (in antiparallel and parallel directions) of stamped kinesinnanotracks. The location of the tracks is inferred from a fluorescent micrograph of the kinesin-decorated template microtubules before walk-off (shown in green). Real-time images of motile transport microtubules (labelled with rhodamine, shown in red) are superimposed. Playback of the movies 10x sped up.

S3 Cargo transport along stamped kinesin nanotracks. The location of the tracks is inferred from a fluorescent micrograph of the kinesin-decorated template microtubules before walk-off (shown in red). Real-time images of streptavidin coated quantum dots (shown in green) that were bound to motile transport microtubules (labelled with rhodamine and biotin, not shown) are superimposed. Playback of the movie 20x sped up.

S4 Guided motility of a brightly labelled (rhodamine) transport microtubule along a biotinylated kinesin track formed 'on top' of a dimly labelled (rhodamine) template microtubule. The template microtubule was immobilized on the surface using tubulin antibodies. Playback of the movie 7.5x sped up.

S5 Guided motility of Alexa-488 labelled transport microtubules along biotinylated kinesin tracks formed 'on top' of template microtubules. The kinesin molecules were linked to the template microtubule, which was immobilized on the surface with rhodamine antibodies, using fluorescein-labelled streptavidin. Playback of the movie 10x sped up.

S6 Guided motility of brightly labelled (rhodamine) transport microtubules along Ncd tracks formed 'on top' of dimly labelled (rhodamine) template microtubules. Playback of the movie $45 x$ sped up.

S7 Guided motility of brightly labelled (rhodamine) transport microtubules along Ncd tracks formed 'on top' of dimly labelled (rhodamine) template microtubules. The transport microtubule takes a turn at the Ncd track crossing and gets redirected. Playback of the movie 30x sped up.

S8 Guided motility of brightly labelled (rhodamine) transport microtubules along Ncd tracks formed 'on top' of dimly labelled (rhodamine) template microtubules. Two transport microtubules are observed sliding contemporaneously on one Ncd track in opposite directions. Playback of the movie 30x sped up. 